Original Article

Genomics and Bioinformatics

\title{
The combined analysis as the best strategy for Dual RNA-Seq mapping
}

\author{
Eliandro Espindula ${ }^{1}$, Edilena Reis Sperb ${ }^{1}$, Evelise Bach ${ }^{1}$ and Luciane Maria Pereira Passaglia ${ }^{1}$ iD \\ ${ }^{1}$ Universidade Federal do Rio Grande do Sul (UFRGS), Instituto de Biociências, Departamento de Genética, \\ Porto Alegre, RS, Brazil.
}

\begin{abstract}
In Dual RNA-Seq experiments the simultaneous extraction of RNA and analysis of gene expression data from both interacting organisms could be a challenge. One alternative is separating the reads during in silico data analysis. There are two main mapping methods used: sequential and combined. Here we present a combined approach in which the libraries were aligned to a concatenated genome to sort the reads before mapping them to the respective annotated genomes. A comparison of this method with the sequential analysis was performed. Two RNA-Seq libraries available in public databases consisting of a eukaryotic (Zea mays) and a prokaryotic (Herbaspirillum seropediceae) organisms were mixed to simulate a Dual RNA-Seq experiment. Libraries from real Dual RNA-Seq experiments were also used. The sequential analysis consistently attributed more reads to the first reference genome used in the analysis (due to cross-mapping) than the combined approach. More importantly, the combined analysis resulted in lower numbers of cross-mapped reads. Our results highlight the necessity of combining the reference genomes to sort reads previously to the counting step to avoid losing information in Dual RNA-Seq experiments. Since most studies first map the RNA-Seq libraries to the eukaryotic genome, much prokaryotic information has probably been lost.
\end{abstract}

Keywords: Dual RNA-Seq, sequential analysis, combined analysis, mapping strategies.

Received: July 02, 2019; Accepted: November 26, 2019.

\section{Introduction}

Organisms modulate their gene expression in order to establish many interactions, from pathogenic to beneficial relationships (Wolf et al., 2018). There is a myriad of eukaryotic-prokaryotic interaction systems being studied, mainly focusing on pathogens and host gene expression responses, and pathogen-associated molecular patterns (PAMPs) (Westermann et al., 2012). Besides that, another successful molecular interaction being widely studied is the relationship between plants and beneficial plant growth promoting bacteria (PGPB), which finds application in the understanding of agricultural inoculants (Balsanelli et al., 2016, Bruto et al., 2014, Camilios-Neto et al., 2014).

Changes in gene expression or transcriptomes were first studied by microarray experiments focusing on only one of the interacting organisms (Barret et al., 2009, Mela et al., 2011). Recently, the RNA sequencing methodology (RNA-Seq) constitutes a promising approach for the parallel study of both interacting organisms, which was called Dual RNA-Seq (Westermann et al., 2012). In the beginning, this technique presented some restrictions related to

Send correspondence to Luciane Maria Pereira Passaglia. Universidade Federal do Rio Grande do Sul (UFRGS), Instituto de Biociências, Av. Bento Gonçalves 9500, prédio 43312, sala 207b, 91501-970 Porto Alegre, RS, Brazil. E-mail: luciane.passaglia@ufrgs.br cost and a significant amount of data management, which is being surpassed by the advent of new sequencing methodologies and bioinformatic tools. However, many RNA-Seq experiments still focused in only one organism of the interaction (Hegedüs et al., 2009, Boscari et al., 2013, Pankievicz et al., 2016, Verwaaijen et al., 2017), whereas others assessed the transcriptome of both interacting organisms (Westermann et al., 2012, Choi et al, 2014, Aprianto et al., 2016, Westermann et al., 2016, Reeder et al., 2017, Westermann and Vogel 2018, Wolf et al., 2018).

To perform a Dual RNA-Seq, steps of RNA isolation from both organisms, rRNA depletion, and cDNA library construction were adapted from the ones applied to simple RNA-Seq experiments (Westermann et al., 2012). To analyze Dual RNA-Seq data, there are two approaches to choose: sequential or combined analysis (Wolf et al., 2018). As the names say, the former consists of the sequential analysis of the libraries against the reference genomes, one after the other (Camilios-Neto et al., 2014). In this approach, reads that fail to map to the first chosen reference genome are assumed to belong to the second genome. Therefore, these unmapped reads are the only ones used to map to the second genome (Packard et al., 2017, Verwaaijen et al., 2017). On the other hand, in combined analysis the libraries are aligned to a chimeric reference genome by concatenating the reference genomes (Aprianto et al., 
2016). All reads that aligned equally well to both genomes or have low alignment accuracy are removed.

Even though both methodologies described above are used to analyze Dual RNA-Seq data, they apparently are simple adaptations from the RNA-Seq methodologies that analyze one transcriptome at the time (Förstner et al., 2014, Westermann et al., 2016) and it seems critical to compare and evaluate which is the best choice for Dual RNA-Seq experiments. It is also worth considering that there is no consensus about the use or not of sequences that can align in more than one genome. Even though simultaneous read mapping has been suggested in 2012 (Westermann et al., 2012), most of the Dual RNA-Seq works still opt to use the sequential approach (Kovalchuk et al., 2019; LaMonte et al., 2019; Mateus et al., 2019; Montoya et al., 2019; Mutha et al., 2019).

Here we present a mapping strategy for the combined analysis that consists of: i) aligning the Dual RNA-Seq libraries against a single file containing both reference genomes; ii) after this first mapping procedure, the reads attributed to each genome are extracted and saved into separated files; iii) these files are then used as individual libraries for the counting step using the respective annotated genome. Besides that, we present comparisons of this methodology to the sequential analysis to emphasize the importance of carefully choosing the mapping strategies for Dual RNA-Seq analysis. We test our approach using RNA-Seq libraries from different interaction systems. In two of them, we used data available in public databases, whereas in another analysis, the RNA-Seq libraries were part of an experiment performed in our laboratory that aimed to study the interaction between Glycine max roots and the bacterium Bradyrhizobium elkanii.

\section{Material and Methods}

\section{RNA-Seq libraries and reference genomes}

In order to test the combined analysis, we used RNASeq libraries available in public databases.

Firstly, data from two independent works were used to simulate a Dual RNA-Seq library. These data consisted in: NT-1 and NT-2 libraries from the bacterium Herbaspirillum seropedicae SmR1, available in the ArrayExpress database under the accession number E-MTAB-2842 (Bonato et al., 2016); and four mRNA libraries isolated from the central portion of the starchy endosperm of Zea mays (maize) $\mathrm{cv}$. B73 six days after pollination, available in the NCBI database under the accession number SRP043224 (Thakare et al., 2014). Herbaspirillum and maize libraries were merged into a single file for each organism.

To verify if the results observed using the individual (and the Chimera) libraries are repeated in real Dual RNA-seq experiments, libraries from two Dual RNA-seq experiments were also evaluated. The first dataset was comprised of dual RNA-Seq paired-end data from Lanubile et al. (2014), who investigated maize root genes involved in the defensive response to the infection caused by the fungus Fusarium verticillioides. Libraries of the biological replicates of the susceptible maize variety $\mathrm{CO} 354$ inoculated with $F$. verticillioides were obtained from NCBI, accession numbers SRR1186869, SRR1186870, and SRR1186871 (Lanubile et al., 2014).

The second dataset was obtained from an unpublished experiment performed in our laboratory that consisted of Dual RNA-Seq single-end libraries. The experiment was designed to evaluate the interaction between two varieties of soybean (Glycine max, EMBRAPA 48 and BR 16) with the bacterium Bradyrhizobium elkanii strain SEMIA 587. Libraries were obtained as described below and were deposited at NCBI under the accession numbers: SRR7206486: BR16, replicate I; SRR7206485: BR16, replicate II; SRR7206490: EMBRAPA 48, replicate I; SRR7206489: EMBRAPA 48, replicate II.

Reference genomes of B. elkanii USDA76 (GCF_000379145.1), G. max (GCF_000004515.5), $H$. seropedicae Z67 (GCF_001040945.1), Fusarium verticillioides (GCF_000149555.1) and Z. mays cv. B73 (GCF_000005015.2) and their respective annotations were obtained from NCBI.

\section{Data analysis}

The CLC Genomics Workbench 8.0 (CLC - Bio; QIAGEN) toolkit was used to perform the trimming, mapping, and counting steps. The "Trimming" tool was used to trim reads smaller than 20 nucleotides from the RNA-Seq libraries, according to the program default settings for quality control. The "Convert to Tracks" tool was applied to the reference genomes to correctly associate them to the respective annotations.

Trimmed H. seropedicae and Z. mays RNA-Seq libraries were aligned to their respective reference genomes to eliminate possible contaminant reads, using the "Map to a Reference" tool with the parameters set to 0.8 of minimum length fraction and 0.8 of minimum similarity fraction. This procedure was called direct mapping and the libraries were called filtered libraries (Figure S1A). Both filtered libraries (from Herbaspirillum or maize) were exported as separate fastq files, which were further merged into a single file to form a Chimera Library to simulate a Dual-RNA-Seq experiment (Figure S1B).

We considered cross-mappings the number of reads that belonged to one organism's transcriptome that mapped to the other organism's genome. To check for cross-mapping, each RNA-Seq filtered library was aligned to the reference genome of the other organism (Figure S2A). Both cross-mapping and contamination checking steps were useful to further evaluate our results. The $H$. seropedicae and $Z$. mays reference genomes were also merged into a single file (Combined Reference), and each RNA-Seq filtered li- 
brary from Herbaspirillum and maize was aligned to the Combined Reference file (Figure S2B).

The Chimera Library was used for the sequential and combined analyses and mapping was done with the "Map to a reference" tool of CLC's program. The first sequential analysis was performed aligning reads to the maize reference genome to generate the first set of data (Eukaryote first- Figure 1A). Afterward, the exact opposite was performed, and the reads were mapped against the bacterium reference genome first to produce the second set of data (Prokaryote first- Figure 1B). In the combined analysis, we aligned the Chimera Library to the Combined Reference file to sort out the sequences belonging to one or another genome (Figure 1C). This Combined Reference was made by concatenating the files of the maize and Herbaspirillum reference genomes into a single reference file. For this purpose, we used the command "cat" of the Linux terminal to merge files. After sorting the sequences, those attributed to each genome were extracted and exported as separate fastq files. Files were imported back to CLC to count the reads of each library as described below.

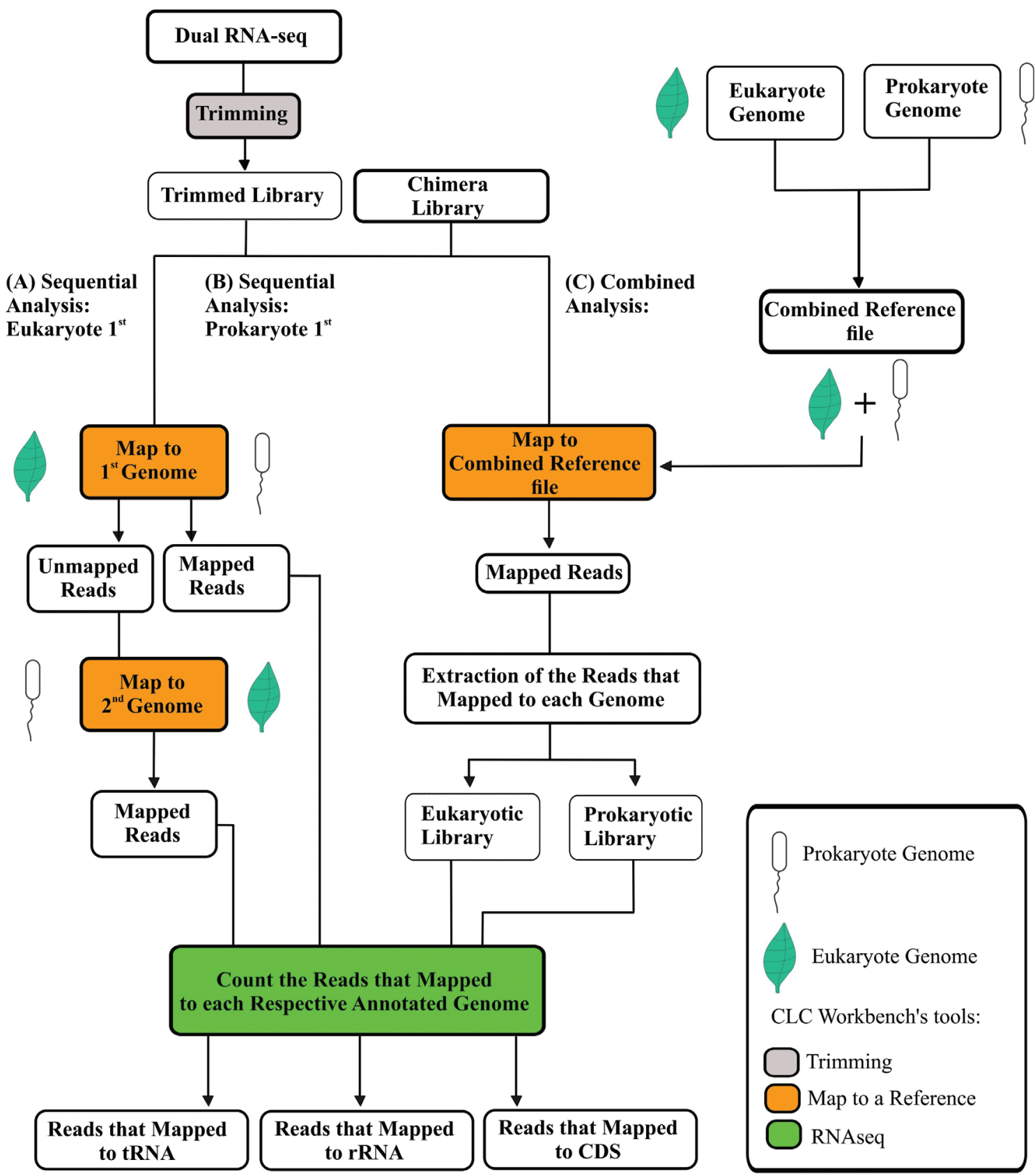

Figure 1 - Mapping strategies for Dual RNA-Seq analysis. (A) Sequential analysis aligning libraries to the eukaryotic genome first- Eukaryote $1^{\text {st }}$; (B) Sequential analysis aligning libraries to the prokaryotic genome first- Prokaryote $1^{\text {st }} ;(\mathrm{C})$ Combined analysis. 
Reads from RNA-Seq libraries of Z. mays, H. seropedicae, and from the Chimera library that aligned to tRNA, rRNA, and to CDS (coding DNA sequence) loci were counted using the CLC's tool "RNAseq" with the parameters set to 0.8 of minimum length fraction and 0.8 of minimum similarity fraction, not mapping to intergenic regions, and allowing a maximum of 5 hits (Figure 1).

To verify if a more rigorous set up condition could improve the results, the analyses were also done using the parameters of 0.9 of minimum length fraction and 0.8 of minimum similarity fraction. This condition is most commonly used for bacterial RNA-seq library alignments (Camilios-Neto et al., 2014, Bonato et al., 2016).

The specificity, sensitivity, accuracy, and precision of each mapping method were also calculated, estimating the true positives, the true negatives, the false positives, and the false negatives reads of each condition. Table S1 details which reads were considered in each group.

In order to compare the results observed for the Chimera Library, the dual RNA-Seq libraries obtained in the soybean/Bradyrhizobium and maize/Fusarium experiments (Lanubile et al., 2014) were also analyzed using the sequential and combined approaches with the parameters of 0.8 of minimum length fraction and 0.8 of minimum similarity fraction. In the maize/Fusarium experiment, some reads were mapped as broken pairs. Although these reads could align independently, none of the possible placements of the pair satisfied the pairing criteria. These reads were then treated as independent and marked as broken pairs. As these reads satisfied the mapping criteria, they were maintained in the following steps of the analysis.

Soybean varieties, bacterial strain, inoculation, growth, and experimental conditions

Soybean (Glycine max) plants of the contrasting genotypes EMBRAPA 48 and BR 16 (Oya et al., 2004) were grown under controlled temperature $\left(26 \pm 4{ }^{\circ} \mathrm{C}\right)$, luminosity $\left(\sim 600 \mu \mathrm{mol} \mathrm{m} \mathrm{m}^{-2} \mathrm{~s}^{-1}\right)$, and photoperiod (18/6 h light/dark). Cultivation was carried out in magenta boxes sealed in the root system, under a hydroponic system. Nutrients were supplied through the Hoagland's nutritive solution strength (Hoagland and Arnon, 1938), which was replaced every three days. The nutritive solution was modified lacking nitrogen to stimulate nodulation. Soybean seeds were surface-sterilized by washing them three times with autoclaved ultrapure water, followed by soaking them in $70 \%$ ethanol for $3 \mathrm{~min}$, and by a solution of $2 \%$ sodium hypochlorite and $2.5 \%$ Tween 20 for $30 \mathrm{~min}$. Seeds were then washed three times with sterile distilled water by gentle shaking (Faleiro et al., 2013). All solutions and materials used were sterilized at $120{ }^{\circ} \mathrm{C}$ for $30 \mathrm{~min}$. When the V2-V3 stage (Fehr et al., 1971) was reached, seedlings were inoculated with the symbiotic bacterium Bradyrhizobium elkanii SEMIA 587. B. elkanii was cultivated in yeast-mannitol liquid medium (Somasegaran and Hoben, 1994) in an or- bital shaker $\left(28^{\circ} \mathrm{C}, 120 \mathrm{rpm}\right)$. When cultures reached an $\mathrm{OD}_{600}$ of 0.6 , they were collected and centrifuged for 10 $\min$ at $10,000 \mathrm{x} g$ at $4{ }^{\circ} \mathrm{C}$. The resulting pellets were washed twice with sterile $0.85 \% \mathrm{NaCl}$ solution, suspended in the same solution, and then diluted to obtain the inoculation solution at a concentration of approximately $10^{8} \mathrm{CFU} / \mathrm{mL}$ (colony forming units).

Inoculation of the roots was performed by submerging them into the inoculant solution for $60 \mathrm{~s}$. Inoculated roots were immediately frozen in liquid nitrogen and cryopreserved at $-80{ }^{\circ} \mathrm{C}$ for subsequent RNA isolation. Two biological replicates composed of pooled root seedlings from five plants were used for each genotype, resulting in four composed samples for further RNA isolation.

RNA isolation, mRNA enrichment, cDNA synthesis, and sequencing

Total RNA isolation of $G$. max root seedlings inoculated with B. elkanii was done using TRIzol (Invitrogen) reagent. The integrity of RNA was verified on $1.5 \%$ agarose gel. Concentration and purity were determined by spectrophotometry at $260 \mathrm{~nm}$ and $280 \mathrm{~nm}$ (Jahn et al., 2008) measured in Nanodrop LITE spectrophotometer (Thermo Fisher Scientific). RNA samples were subjected to a purification step using PureLink RNA Micro kit (Ambion), treated with DNaseI (Invitrogen) and then rRNA was depleted using the RiboMinus Plant Kit for RNA-Seq (Invitrogen). The cDNA libraries were constructed using the Ion total RNA-Seq kit v2 for Whole Transcriptome Library. All RNA quantification and quality evaluation were performed at the Bioanalyzer - Agilent 2100 instrument. Each cDNA library obtained was sequenced using the Ion PI Template OT2 200 Kit v3 and the Ion PI Sequencing 200 Kit v3 at the IonTorrent ${ }^{\circledR}$ platform (Thermo Fisher Scientific). All kits and reagents were used according to manufacturer's instructions.

The presence of the bacterium in plant roots was subsequently determined by the detection of its $16 \mathrm{~S}$ rRNA gene sequences in the transcriptome library.

\section{Results}

\section{Data analysis using independent RNA-Seq libraries}

Before starting the analysis, trimmed RNA-Seq libraries from $Z$. mays and $H$. seropedicae were filtered by direct mapping to each genome to avoid potential contamination sequences (Figure S1A). After filtering, the $H$. seropedicae RNA-Seq library presented approximately 44 million reads, while the Z. mays RNA-Seq library presented approximately 22 million reads. The Chimera Library, which simulates a Dual RNA-Seq experiment, was constructed joining these two libraries (Figure S1B) and presented approximately 66 million reads (Table 1).

Cross-mappings were determined by the number of reads from one organism RNA-Seq library that could be at- 


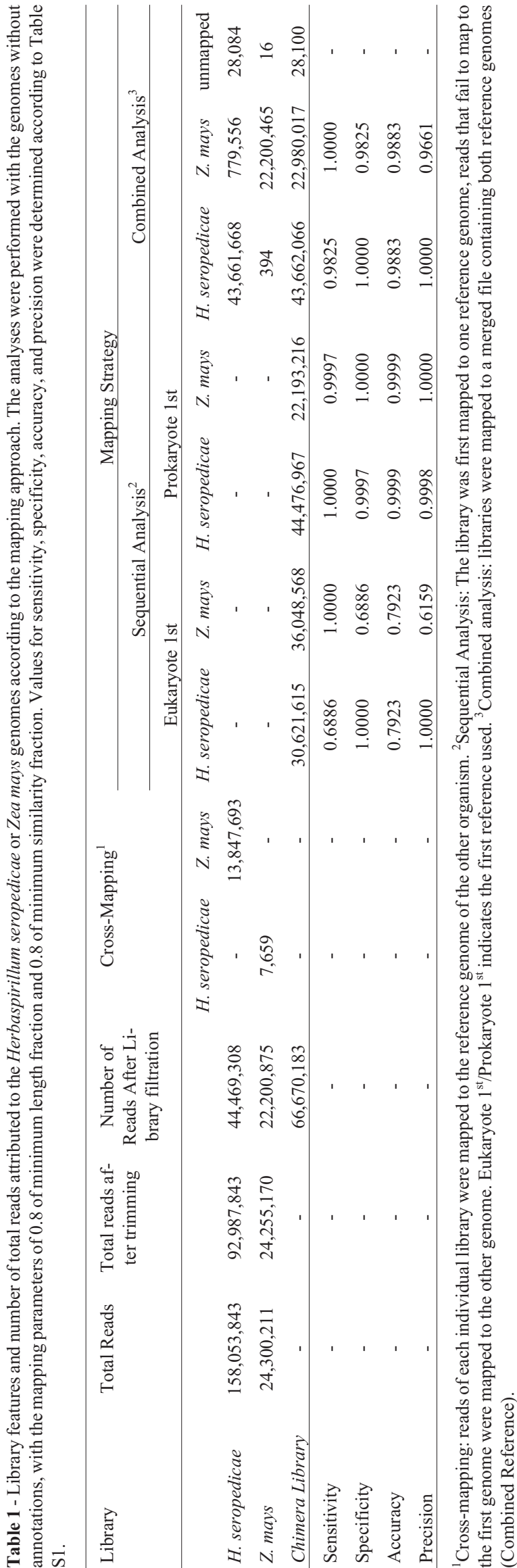

tributed to the other organism reference genome (Figure S2A). Interestingly, approximately 13 million reads from the $H$. seropedicae RNA-Seq library aligned to the $Z$. mays genome, while 7,659 reads from the $Z$. mays RNA-Seq library mapped to the $H$. seropedicae genome (Table 1 ). On the other hand, when we mapped the individual RNA-Seq libraries to the Combined Reference file (Figure S2B), which was constructed by concatenating $H$. seropedicae and $Z$. mays genomes (Figure 1), more surprising results were obtained. When the H. seropedicae RNA-Seq library was aligned to the Combined Reference file, approximately 43 million reads were attributed to $H$. seropedicae genome and 779,556 reads to $Z$. mays genome; whereas when $Z$. mays RNA-Seq library was mapped to the Combined Reference file, 394 reads were attributed to $H$. seropedicae genome and approximately 22 million reads to $Z$. mays genome (Table 1). These results showed that even in the presence of both reference genomes some reads still mapped incorrectly, although the numbers of reads incorrectly aligned were much smaller than the numbers of crossmapping reads obtained when one RNA-Seq library was aligned to the genome of the other organism.

After estimating cross-mappings, we evaluated both the sequential and the combined approach of Dual RNASeq analysis. The sequential analysis consisted of aligning the Chimera Library to one reference genome before the other. Reads that aligned to the first genome constituted this organism's library. Unmapped reads are then mapped to the second reference genome. All reads that aligned to the second genome comprised this organism's library. We first mapped the Chimera Library to Z. mays reference genome, and then the unmapped reads were mapped to the H. seropedicae reference genome (Figure 1A). Approximately 30 million reads were attributed to $H$. seropedicae genome, and approximately 36 million reads were attributed to $Z$. mays genome (Table 1 - Eukaryote $1^{\text {st }}$ ). When we did the opposite and first mapped the Chimera Library to the $H$. seropedicae reference genome (Figure 1B), approximately 44 million reads were attributed to $H$. seropedicae genome and approximately 22 million reads to $Z$. mays genome (Table 1 - Prokaryote $1^{\text {st }}$ ).

Finally, we performed the combined analysis that consists of aligning the RNA-Seq library to a file containing a combination of reference genomes (Combined Reference). We mapped the Chimera Library to the Combined Reference file, and this alignment approach attributed approximately 43.6 million reads to $H$. seropedicae genome and approximately 22.9 million reads to $Z$. mays genome (Table 1 - Combined analysis, Figure 1C). After the mapping procedure, reads attributed to each genome were extracted, saved into separated files (Figure S3), and used as individual libraries for the counting step (Figure 1C). All reads that aligned to $H$. seropedicae or $Z$. mays genomes were counted using the corresponding reference genome and its respective annotations (Tables 2 and 3 ). In all counts 
we observed unmapped reads. This is likely due to the parameters chosen for counting the reads in the CLC's "RNAseq" tool, as reads that mapped in more than five loci or mapped in intergenic regions were excluded.

The counting of reads that aligned to the respective genome in the direct, sequential, and combined analysis showed interesting results (Table 2). In the direct analysis, for the H. seropedicae RNA-Seq library, 1,423,990 reads were attributed to tRNA, 31,630,385 reads to rRNA, and 9,00,409 (unique mapped) reads to CDS loci using the $H$. seropedicae genome, 616,765 remained unmapped, while for Z. mays RNA-Seq library, we counted 1,692 tRNA reads, 3,003 rRNA reads, and 21,051,646 CDS loci reads using the $Z$. mays genome, 1,144,534 remained unmapped (Table 2 - Direct mapping). In the sequential analysis, when we first mapped the Chimera Library to the $Z$. mays genome, we counted using the genome of $H$. seropedicae $1,181,068$ tRNA reads, 21,550,448 rRNA reads, 6,052,838 (unique mapped) CDS loci reads, and 1,805,595 reads remained unmapped, while when using the genome of $Z$. mays, we counted 89,216 tRNA reads, 3,254,994 rRNA, 24,843,247 (unique mapped) CDS loci reads, and $5,270,069$ reads remained unmapped (Table 2 - Eukaryote $\left.1^{\text {st }}\right)$. On the other hand, when we first mapped the Chimera Library to the $H$. seropedicae genome and also counted with $H$. seropedicae files, 1,423,992 reads were attributed to tRNA, 31,631,168 reads to rRNA, 9,010,911 (unique mapped) to CDS loci, and 2,330,780 reads remained unmapped, while when counting using the $Z$. mays genome, 1,686 reads were attributed to tRNA, 2,255 reads to rRNA, 20,157,930 (unique mapped) reads to CDS loci, and $1,144,183$ reads remained unmapped (Table 2 - Prokaryote $\left.1^{\text {st }}\right)$. Finally, when we counted the reads that mapped to each reference genome using the combined analysis and counted using $H$. seropedicae files, 1,419,674 reads were attributed to tRNA, 31,304,115 reads to rRNA, 8,591,366 (unique mapped) reads to CDS loci, and 2,287,572 reads remained unmapped, while when counting with the $Z$. mays genome, 1,971 reads were attributed to tRNA, 79,917 reads to rRNA, 20,530,853 (unique mapped) reads to CDS loci, and 1,315,273 reads remained unmapped (Table 2 - Combined analysis).

The amount of multi-mapped reads assigned to CDS loci was also evaluated. We observed that at least $4 \%$ of the reads attributed to maize CDS are multi-mapped reads. For Herbaspirillum this amount corresponded to less than 1\% of the total reads attributed to Herbaspirillum CDS (Table 2).

All the analyses described above were also performed using 0.9 of minimum length fraction and 0.8 of minimum similarity fraction as a more stringent parameter. After the filtering step, the H. seropedicae RNA-Seq library presented approximately 41 million reads, while the Z. mays RNA-Seq library presented approximately 21 million reads. The Chimera Library presented approximately 62

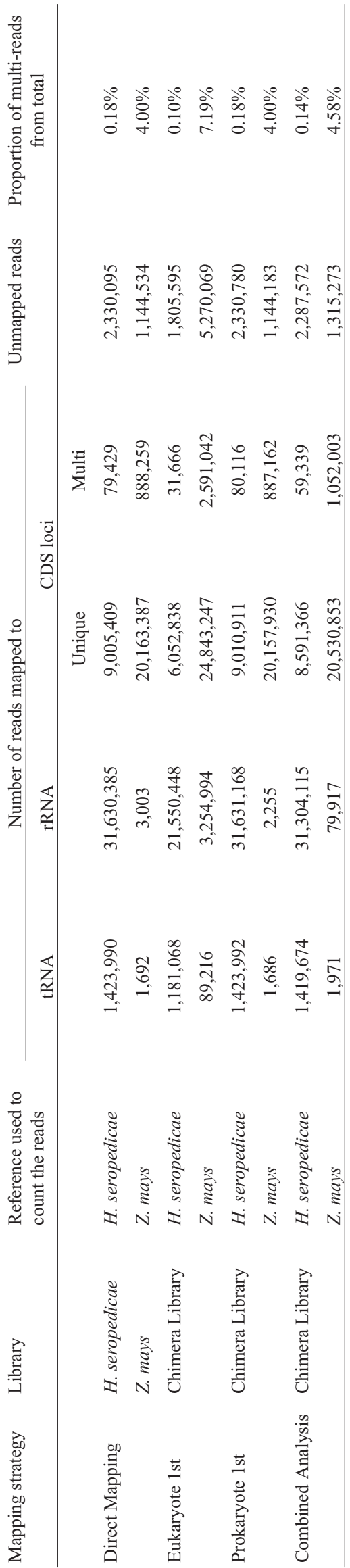


Table 3 - Comparison of the number of reads incorrectly mapped due to cross-mapping, with the mapping parameters of 0.8 of minimum length fraction and 0.8 of minimum similarity fraction. Reads that incorrectly mapped to the reference genome were counted using the annotated genome indicated on the table. The unmapped reads are a result of the counting parameters that eliminate reads that mapped in more than five loci and of the intergenic regions.

\begin{tabular}{|c|c|c|c|c|c|c|c|}
\hline \multirow[t]{2}{*}{ Library } & \multirow{2}{*}{$\begin{array}{l}\text { Reference Used to Map } \\
\text { the Reads }\end{array}$} & \multirow{2}{*}{$\begin{array}{l}\text { Reference Used to Count the } \\
\text { Cross-Mapped Reads }\end{array}$} & \multicolumn{3}{|c|}{ Number of Reads Mapped to } & \multirow[t]{2}{*}{ CDS* } & \multirow{2}{*}{$\begin{array}{l}\text { Unmapped } \\
\text { reads }\end{array}$} \\
\hline & & & tRNA & rRNA & CDS Loci & & \\
\hline \multirow[t]{4}{*}{ H. seropedicae } & Z. mays & H. seropedicae & 242,922 & $10,079,937$ & $3,000,334$ & 4,553 & 524,500 \\
\hline & & Z. mays & 87,524 & $3,251,991$ & $6,382,643$ & 23,216 & $4,125,535$ \\
\hline & Combined Reference & H. seropedicae & 4,156 & 320,514 & 413,822 & 3,299 & 41,064 \\
\hline & & Z. mays & 279 & 77,231 & 531,276 & 3,298 & 170,770 \\
\hline \multirow[t]{4}{*}{ Z. mays } & H. seropedicae & Z. mays & 6 & 748 & 6,554 & 72 & 351 \\
\hline & & H. seropedicae & 2 & 783 & 6,189 & 65 & 685 \\
\hline & Combined Reference & Z. mays & 0 & 308 & 57 & 43 & 29 \\
\hline & & H. seropedicae & 0 & 329 & 59 & 49 & 6 \\
\hline
\end{tabular}

*CDS with at least 10 reads assigned to them. Exception made to the Z. mays library mapped against the Combined Reference, which refers to CDS with at least one read assigned to it.

million reads (Table S2). Comparing with the amount that was mapped to Herbaspirillum genome using the previous set up parameters, the amount of reads mapped to the Herbaspirillum genome was reduced in 3,137,800. After that, we determined the amount of reads that could crossmap. We highlight the fact that approximately 8.9 million reads from Herbaspirillum mapped to the Zea mays genome (Table S2), which represents a reduction of around $10 \%$ in the number of cross mappings reads. This reduction was probably caused by the reduction of mapped reads observed in the direct mapping. In the counting step we highlight the fact that there was a reduction in the number of identified CDS loci in almost all situations (data not show). For the remaining results, the observed patterns were the same of those observed for the previous set up (Table S3).

In order to investigate the reads that were incorrectly aligned (cross-mapped reads), those reads were also counted using both the respective and the incorrect reference genomes (Figure S2, Tables 3 and S4). When the parameters of 0.8 of minimum length fraction and 0.8 of minimum similarity fraction were applied, although several reads incorrectly mapped to rRNA and tRNA loci, the most important result was that about six million $H$. seropedicae reads were incorrectly attributed to almost 23,216 Z. mays CDS. A similar situation, although with minor effects, was observed for the Z. mays library, where 6,189 reads were incorrectly attributed to $65 \mathrm{H}$. seropedicae CDS. In these cases, only CDS that received at least ten reads assigned to them were considered. When the combined reference file was used, the numbers of reads incorrectly mapped decreased significantly, in particular for the $H$. seropedicae genome. In this case, 531,276 reads from $H$. seropedicae were incorrectly attributed to 3,298 Z. mays CDS. These analyses were also performed using the parameter of 0.9 of minimum length fraction and 0.8 of minimum similarity fraction. For these analyses all the results presented the same pattern observed for the previous parameter, in which the reads that cross mapped were mostly assigned to rRNA and CDS loci (Table S4).

We also estimated the sensitivity, specificity, precision, and accuracy (Table S1) of the methodologies for all parameters tested (Tables 1 and S2). Regardless of the parameter used, we observed that in the sequential analysis Eukaryote $1^{\text {st }}$ the accuracy was lower than in the sequential analysis - Prokaryote $1^{\text {st }}$ or in the Combined Analysis (Tables 1 and S2). When comparing the values of these parameters for the sequential analysis - Prokaryote $1^{\text {st }}$ with those obtained for the Combined analysis, we observed that the accuracy values in both methodologies were equivalent for both parameters, with a slight increase when the more rigorous parameter was used. Taking all this together and to the fact that the mapping parameter of 0.9 of minimum length fraction and 0.8 of minimum similarity fraction lead to a reduction in the number of CDS loci identified (probably caused by the reduction of the amount of reads mapped in the direct mapping), the Combined Analysis with the parameters of 0.8 of minimum length fraction and 0.8 of minimum similarity fraction was used in the following analyses.

Tables S5 and S6 present the top 20 most counted loci among the cross-mapped reads. Table S5 shows the loci where the reads should be aligned in the correct genome, while Table S6 shows the loci where the reads aligned in the incorrect genome. It is interesting to note that most of the incorrectly mapped reads corresponded to genes that code for proteins with different functions, such as kinases, phosphatases, and ribosomal proteins. Several genes coding for hypothetical or uncharacterized proteins were also identified.

\section{Analyses of experimentally obtained Dual RNA-Seq libraries}

The combined Dual RNA-Seq analysis was also applied to RNA-Seq libraries obtained from two experiments, one performed in our laboratory and another carried out by Lanubile et al. (2014). The first one aimed to evaluate the 
interaction of two varieties of $G$. $\max$ with the symbiotic bacterium B. elkanii. The presence of the bacterium in the plant's roots was confirmed by the detection of its $16 \mathrm{~S}$ rRNA gene sequences in the RNA-Seq libraries (data not shown). RNA-Seq libraries obtained from both organisms showed enough quality and coverage to perform gene expression analysis (Table $\mathrm{S} 7$ ). After the trimming procedure, RNA-Seq libraries from the G. max - B. elkanii experiment presented approximately 5 to 9 million reads (Table S7). The Dual RNA-Seq alignment strategies showed that numbers attributed to the eukaryotic genome roughly did not vary among sequential or combined analyses, regardless of the soybean variety used (Figure 2A, Table S7). However, some variation was observed in the number of reads mapped to the prokaryotic genome depending on the mapping approach.

When RNA-Seq reads were first aligned to the eukaryotic genome (Table S7 - Sequential analysis- Eukaryote $1^{\text {st }}$ ), the number of reads attributed to the bacterium was less than $2 \%$ of the total amount of reads (Figure 2B) for both soybean varieties. However, when the opposite analysis was performed (Table S7 - Sequential analysis- Prokaryote $1^{\text {st }}$ ), the number of reads aligned to the prokaryote genome increased significantly, reaching more than $3 \%$ of the total amount of reads mapped in both samples (Figure 2B), also for both soybean varieties. Using the combined analysis,

\section{A - Bradyrhizobium elkanii}

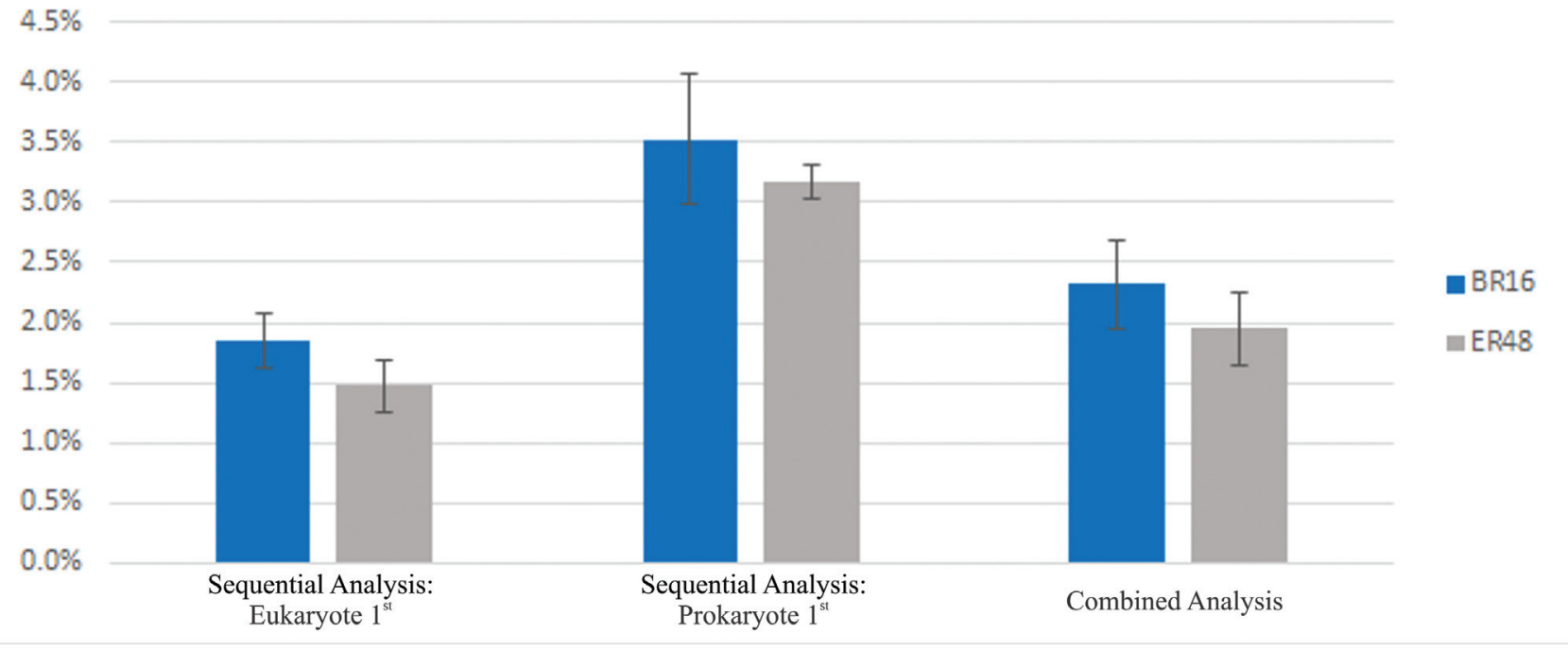

\section{B - Glycine $\max$}

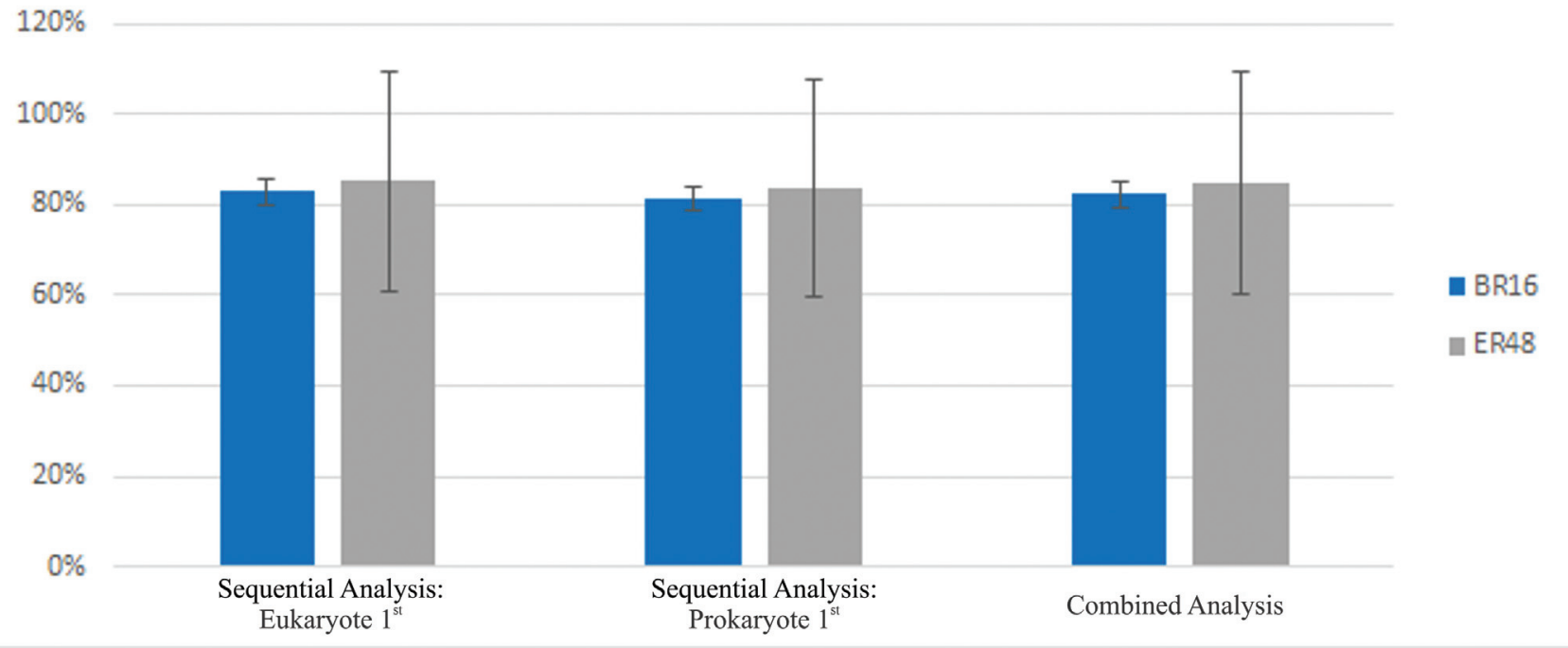

Figure 2 - Percentage of reads mapped to (A) Bradyrhizobium elkanii or (B) Glycine max depending on the methodology used in the Glycine max Bradyrhizobium elkanii experiment. Bars indicate twice the Standard Error. BR16 and ER48: soybean varieties BR16 and Embrapa 48, respectively. 
when reads were aligned to both genomes at the same time, intermediary numbers of reads were attributed to the prokaryote genome, regardless of the soybean variety used (Table S7 - Combined analysis, Figure 2B). The average number of reads attributed to the prokaryote in the combined analysis was not significantly different from the average number attributed at the Sequential Analysis - Eukaryote $1^{\text {st }}$. Despite this fact, the results still indicated that probably some reads that mapped to the first genome used in the sequential approach very likely belong to the second genome and incorrectly mapped to the first genome because the second was not present in the analysis.

The second experiment used to evaluate the combined Dual RNA-Seq analysis was performed by Lanubile et al. (2014), who investigated maize roots gene expression during Fusarium verticillioides infection. Although these authors investigated $Z$. mays genes only, library preparation involves the isolation of mRNAs using poly(A)-tails, which potentially included fungus mRNA. Thus, we chose this library as another example of plant-microorganism interaction. After the trimming procedure, the libraries had from 74 to 83 million reads (Table S8). In this analysis, even though the numbers of reads attributed to both genomes varied according to the previous experiments (Table S8), the average number of reads attributed to each genome according to the methodology used were not significantly different, since the standard errors were substantial (Figure $3)$. Nevertheless, the combined analysis showed an intermediate amount of reads attributed to each genome in comparison with the number of reads observed in the sequential analyses (Table S8, Figure 3), which was similar to the previous analyses.

We also evaluated the amount of multi-mapped reads attributed to CDS loci in both experiments. For the Bradyrhizobium-Glycine max experiment, we observed that more than $60 \%$ of the reads attributed to G. $\max$ CDS were multi-mapped reads. For the Bradyrhizobium we observed that less than $1 \%$ of the reads attributed to CDS loci were multi-mapped reads. In the Fusarium-maize experiment around $7 \%$ of the reads attributed to CDS loci in both organisms were multi-mapped reads (Table 4).

\section{Discussion}

RNA sequencing methodologies are revolutionizing the way we study gene expression. Unlike microarrays, to perform an RNA-Seq analysis there is no need for previous knowledge about the organism. Another advantage of RNA-Seq is that it enables global gene expression analysis since it allows access to different populations of RNA sequences from the organism (Wang et al., 2009, Oshlack et al., 2010). In the last decade, this technique was used to assess gene expression of many organisms and it has recently started to be used to assess the transcriptomes of interacting organisms, called Dual RNA-Seq (Camilios-Neto et al.,
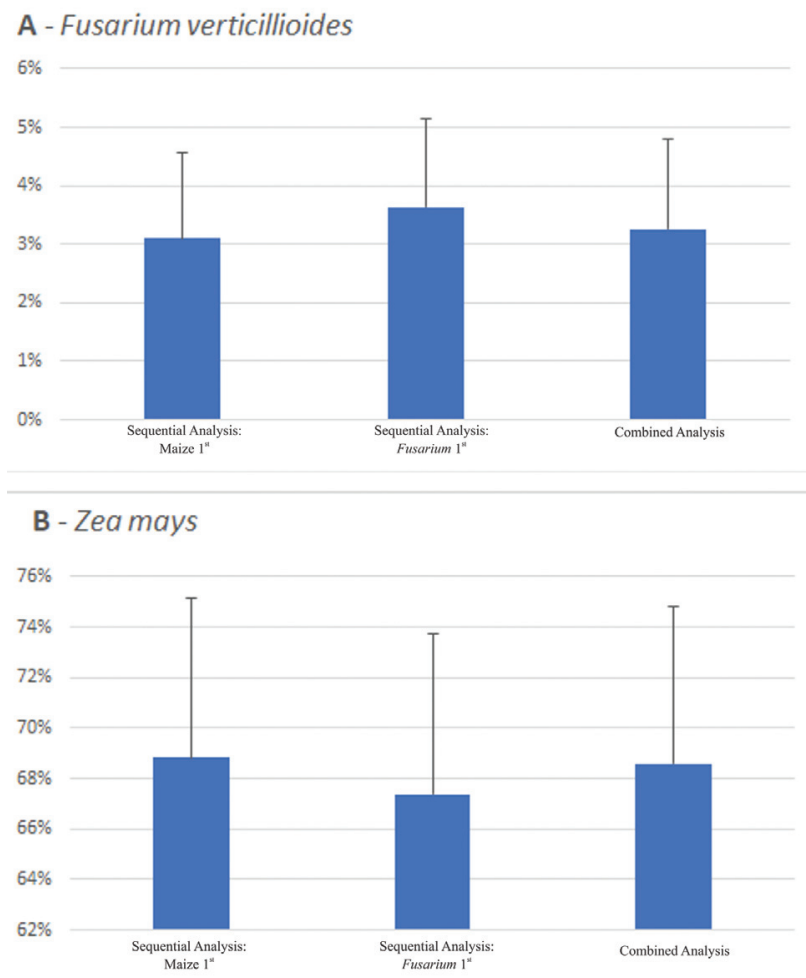

Figure 3 - Percentage of reads mapped to (A) Fusarium verticillioides or (B) Zea mays depending on the methodology used in the Zea mays -Fusarium verticillioides experiment. Bars indicate twice the Standard Error.

2014, Hayden et al., 2014, Baddal et al., 2015, Pankievicz et al., 2016, Westermann et al., 2016).

Despite the difficulties in obtaining libraries containing RNAs from both interacting organisms, there are also problems in sorting the reads in silico. The sequential approach seems to be the most common mapping method chosen, and the order of the genomes used in the analysis is chosen according to study interests (Camilios-Neto et al., 2014, Baddal et al., 2015, LaMonte et al., 2019, Mateus et al., 2019, Montoya et al., 2019). Sometimes the reads of one of the interacting organisms are not considered for the study and are discarded from the analysis (Lanubile et al., 2014, Packard et al., 2017, Verwaaijen et al., 2017). Similarly, reads that aligned equally well to either genome or simply cross-mapped are also sometimes discarded (Baddal et al., 2015, Westermann et al., 2016, Westermann and Vogel, 2018).

Here we used a Combined Analysis, which consists in using a Combined Reference file formed by merging the reference genomes files of both organisms to in silico sort the reads that align to each genome. Once identified, they were extracted and saved in separated files (Figure S3). The libraries formed by the reads of each organism were then counted using the corresponding reference genome with their own annotations. To perform these analyses, we used the CLC's tools set with the parameters usually used to map eukaryotic libraries (Camilios-Neto et al., 2014). 
Table 4 - Number of reads mapped to tRNA, rRNA, and coding loci (CDS) according to the mapping methodology, with the mapping parameters of 0.8 of minimum length fraction and 0.8 of minimum similarity fraction, and experiment used. BR16 and ER48: soybean varieties BR16 and Embrapa 48, respectively. CO354: susceptible maize variety CO354 inoculated with $F$. verticillioides from Lanubile et al. (2014).

\begin{tabular}{|c|c|c|c|c|c|c|c|c|c|}
\hline \multirow{3}{*}{$\begin{array}{l}\text { Samples } \\
\text { Soybean } \\
\end{array}$} & \multirow{3}{*}{$\begin{array}{l}\text { Biological Repeti- } \\
\text { tion } \\
+ \text { Bradyrhizobium: }\end{array}$} & \multirow{3}{*}{$\begin{array}{l}\text { Mapping } \\
\text { Strategy }\end{array}$} & \multirow{3}{*}{$\begin{array}{l}\text { Reference Used } \\
\text { to Count the } \\
\text { Reads }\end{array}$} & \multicolumn{4}{|c|}{ Number of Reads Mapped to } & \multirow{3}{*}{$\begin{array}{l}\text { Un- } \\
\text { mapped } \\
\text { reads }\end{array}$} & \multirow{3}{*}{$\begin{array}{c}\text { Proportion of } \\
\text { Multireads } \\
\text { from total } \\
\end{array}$} \\
\hline & & & & \multirow[t]{2}{*}{ tRNA } & \multirow[t]{2}{*}{ rRNA } & \multicolumn{2}{|c|}{ CDS loci } & & \\
\hline & & & & & & Unique & Multi & & \\
\hline \multirow[t]{4}{*}{ BR16 } & I & Eukaryote $1^{\text {st }}$ & G. $\max$ & 9,262 & 458,414 & $1,386,492$ & $5,505,200$ & 275,078 & $72.11 \%$ \\
\hline & II & & G. $\max$ & 14,526 & 524,794 & $1,553,300$ & $2,218,202$ & 298,956 & $48.12 \%$ \\
\hline & I & & B. elkanii & 6,140 & 137,368 & 18,423 & 408 & 1,349 & $0.25 \%$ \\
\hline & II & & B. elkanii & 8,431 & 153,677 & 20,804 & 318 & 2,025 & $0.17 \%$ \\
\hline \multirow[t]{4}{*}{ ER48 } & I & & G. $\max$ & 7,486 & 284,116 & 853,250 & $34,198,215$ & 161,811 & $96.32 \%$ \\
\hline & II & & G. $\max$ & 12,566 & 400,613 & $1,496,084$ & $4,115,974$ & 263,977 & $65.44 \%$ \\
\hline & I & & B. elkanii & 5,423 & 87,283 & 8,217 & 194 & 1,057 & $0.19 \%$ \\
\hline & II & & B. elkanii & 5,219 & 64,293 & 16,609 & 524 & 1,401 & $0.60 \%$ \\
\hline \multirow[t]{4}{*}{ BR16 } & I & Prokaryote $1^{\text {st }}$ & G. $\max$ & 7,784 & 337,378 & $1,381,270$ & $5,493,766$ & 271,697 & $73.33 \%$ \\
\hline & II & & G. $\max$ & 11,509 & 383,520 & $1,547,288$ & $5,502,769$ & 293,615 & $71.11 \%$ \\
\hline & I & & B. elkanii & 8,597 & 262,024 & 30,995 & 919 & 3,704 & $0.30 \%$ \\
\hline & II & & B. elkanii & 13,152 & 301,072 & 34,724 & 1,183 & 6,201 & $0.33 \%$ \\
\hline \multirow[t]{4}{*}{ ER48 } & I & & G. $\max$ & 5,803 & 209,395 & 848,055 & $3,406,326$ & 158,528 & $73.60 \%$ \\
\hline & II & & G. $\max$ & 10,145 & 321,792 & $1,485,462$ & $4,091,851$ & 258,616 & $66.34 \%$ \\
\hline & I & & B. elkanii & 8,443 & 165,541 & 20,871 & 703 & 4,387 & $0.35 \%$ \\
\hline & II & & B. elkanii & 9,329 & 147,323 & 43,393 & 2,016 & 7,363 & $0.96 \%$ \\
\hline \multirow[t]{4}{*}{ BR16 } & I & Combined & G. $\max$ & 8,571 & 420,015 & $1,384,623$ & $5,504,514$ & 273,168 & $72.51 \%$ \\
\hline & II & Analysis & G. $\max$ & 13,227 & 477,056 & $1,550,710$ & $5,517,511$ & 295,728 & $70.25 \%$ \\
\hline & I & & B. elkanii & 7,097 & 172,756 & 18,760 & 493 & 1,595 & $0.25 \%$ \\
\hline & II & & B. elkanii & 10,299 & 200,206 & 21,413 & 429 & 2,236 & $0.18 \%$ \\
\hline \multirow[t]{4}{*}{ ER48 } & I & & G. $\max$ & 6,617 & 252,998 & 850,854 & $3,418,620$ & 159,801 & $72.91 \%$ \\
\hline & II & & G. $\max$ & 11,597 & 378,032 & $1,493,400$ & $4,114,632$ & 260,854 & $65.74 \%$ \\
\hline & I & & B. elkanii & 6,907 & 118,911 & 8,806 & 251 & 1,166 & $0.18 \%$ \\
\hline & II & & B. elkanii & 6,872 & 89,370 & 17,570 & 631 & 1,613 & $0.54 \%$ \\
\hline \multicolumn{10}{|c|}{ Maize + Fusarium: } \\
\hline \multirow[t]{6}{*}{$\mathrm{CO} 354$} & I & Maize $1^{\text {st }}$ & Z. mays & 257 & 46,053 & $63,605,211$ & $5,020,302$ & $1,973,035$ & $7.11 \%$ \\
\hline & II & & Z. mays & 280 & 43,537 & $64,060,395$ & $5,074,624$ & $2,015,545$ & $7.13 \%$ \\
\hline & III & & Z. mays & 279 & 36,277 & $55,320,528$ & $4,407,818$ & $1,690,684$ & $7.17 \%$ \\
\hline & I & & F. verticillioides & 47 & 509 & $2,620,154$ & 218,485 & 150,957 & $7.31 \%$ \\
\hline & II & & F. verticillioides & 23 & 293 & $1,424,365$ & 119,595 & 83,640 & $7.35 \%$ \\
\hline & III & & F. verticillioides & 53 & 526 & $3,381,869$ & 282,827 & 196,770 & $7.32 \%$ \\
\hline \multirow[t]{6}{*}{$\mathrm{CO} 354$} & I & Fusarium $1^{\text {st }}$ & Z. mays & 257 & 35,577 & $63,061,802$ & $4,983,688$ & $1,678,420$ & $7.14 \%$ \\
\hline & II & & Z. mays & 280 & 35,190 & $63,559,272$ & $5,042,606$ & $1,713,630$ & $7.17 \%$ \\
\hline & III & & Z. mays & 279 & 26,101 & $54,799,352$ & $4,372,544$ & $1,446,308$ & $7.21 \%$ \\
\hline & I & & F. verticillioides & 48 & 583 & $3,139,676$ & 276,241 & 458,718 & $7.13 \%$ \\
\hline & II & & $F$. verticillioides & 23 & 369 & $1,903,321$ & 170,812 & 396,794 & $6.91 \%$ \\
\hline & III & & F. verticillioides & 53 & 601 & $3,879,192$ & 339,538 & 453,663 & $7.27 \%$ \\
\hline \multirow[t]{6}{*}{$\mathrm{CO} 354$} & I & Combined & Z. mays & 257 & 38,312 & $63,519,081$ & $5,007,715$ & $1,960,084$ & $7.10 \%$ \\
\hline & II & Analysis & Z. mays & 280 & 37,882 & $63,994,094$ & $5,066,322$ & $2,006,113$ & $7.13 \%$ \\
\hline & III & & Z. mays & 279 & 28,336 & $55,232,851$ & $4,393,751$ & $1,677,822$ & $7.16 \%$ \\
\hline & I & & F. verticillioides & 48 & 516 & $2,629,064$ & 221,907 & 166,912 & $7.35 \%$ \\
\hline & II & & F. verticillioides & 23 & 291 & $1,417,789$ & 121,161 & 94,107 & $7.42 \%$ \\
\hline & III & & $F$. verticillioides & 53 & 533 & $3,403,439$ & 287,282 & 213,560 & $7.36 \%$ \\
\hline
\end{tabular}


Before testing the combined approach, we determined the number of cross-mapped reads between the two RNA-Seq libraries using the reference genome of the other organism of the Combined Reference file. After aligning them, the reads that mapped to the incorrect genome (cross-mapped reads) were counted using both the correct and incorrect reference genome. This was done to identify the loci where these reads were aligned in the incorrect genome and the loci where they should be assigned in the correct one (cross-mapping; Tables 3, S7 and S8). Our results showed that the combined analysis consistently assigned a lower number of reads to the incorrect organism due to cross-map, allowing the program to better attribute the reads to its corresponding genome, leading to a lower number of cross-mappings (Table 1 and S2).

After these cross-map evaluations, two sequential analyses were performed, and the obtained results were compared with the results from the combined analysis. For both sequential analyses, it was possible to notice that the first genome used on the mapping step was always benefited. We observed that the first genome used to map the reads received the full number of reads that could crossmap with the genome of the other organism (Table 1 and S2). We also noticed that even though many of the crossmapping reads mapped to rRNA genes, a significant number of cross-mapping reads were attributed to CDS loci in all methodological approaches. However, in the combined analysis, the loss of reads due to cross-mapping was lower than in the sequential analysis (Table 3 and S4). Also interesting was the fact that the $H$. seropedicae genome lost more reads for the $Z$. mays genome due to cross-mapping than the other way around.

The sensitivity, specificity, precision, and accuracy of the methodologies in the different parameters tested were also calculated (Tables 1 and S2). According to our results, regardless of the mapping parameters chosen, the Sequential Analysis - Eukaryote 1st presented the worst results for accuracy and precision. On the other hand, the Sequential Analysis - Prokaryote 1st and the Combined analysis presented equivalent results for accuracy and precision, and a slight increase was achieved with more restrictive mapping parameters (Table S2). As the accuracy and precision of the mapping in the Sequential Analysis directly depends on which organism is first used in the analysis, and as the Combined Analysis presented similar values of accuracy and precision as the Sequential Analysis Prokaryote 1st, we recommend the use of the Combined Analysis since it avoids the tendency of choosing which genome will be the first to be used in the analysis.

To compare the in silico data with real Dual RNASeq samples, libraries from two different Dual RNA-Seq experiments were submitted to both sequential and the combined approaches. In both experiments, the results obtained were similar and followed the results from the in silico data, with the combined analysis showing intermedi- ary values when compared to values attributed by the sequential analyses (Tables S7 and S8). For the G. $\max -B$. elkanii experiment, the average amount of reads attributed to the combined analysis was significantly different only concerning the Sequential - Prokaryote $1^{\text {st }}$ data (Figure 2B). Schurch et al. (2016) recommended that at least three biological replicates must be used in order to detect genes being differentially expressed. Since the G. max - B. elkanii experiment contained only two biological replicates we hypothesized that with more biological replicates these two methodologies should present significant differences concerning the number of reads attributed to each organism.

Another interesting fact was observed in the Lanubile et al. (2014) experiment. When comparing the average amount of reads attributed to each genome, regardless of the methodology used, no significant differences were observed (Figure 3). Analyzing our results, it seems that paired-end sequencing was also useful to make the two eukaryotic genomes more distinguishable and less prone to cross-mappings (Figure 3). Therefore, one should consider using paired-end libraries allied with the combined analysis in order to reduce the number of cross-mappings during Dual RNA-Seq experiments.

Since we detected that a significant number of crossmapping reads aligned to gene coding regions of the genomes, we can assume that this happened because the interacting organisms should have similar metabolic pathways or due to homologous sequences. Eliminating these reads from the libraries before counting them represents a problem because a considerable amount of transcriptional information will be lost. Therefore, all reads that align to both genomes (with different degrees of similarity to each genome) will align to the first genome used in the sequential mapping approach. This might lead to an overestimation of the expressed genes of the first genome used in the sequential mapping method. Similarly, the expressed genes of the second genome might be underestimated. This problem seems to be more critical for those interested in the prokaryotic transcriptome. Prokaryotic RNA is always less abundant in libraries prepared from mixed sources (Westermann et al., 2012); therefore, techniques that underestimate their read counts should be avoided. The combined analysis seems to be more reasonable to avoid these under/overestimations.

Aprianto et al. (2016) suggested a Dual RNA-Seq approach in which they aligned the libraries to a chimeric genome. To create this genome, they concatenated the Streptococcus pneumoniae genome as an extra chromosome of Homo sapiens and adjusted the annotated genomes. All procedures were performed with command-line entries that demands some bioinformatic knowledge and programming skills. Another objective of our work was to describe a way to analyze the Dual RNA-Seq libraries without the need for high computational skills. Therefore, to perform the proposed combined analysis, the CLC Workbench was used. 
This program is user-friendly since it works with a graphic interface and has several internal tutorials, which demands only basic bioinformatics skills. Another aspect, and according to Baruzzo et al. (2017), CLC Workbench, alongside with Novoalign and STAR, is one of the best aligners for eukaryotes in use nowadays, even when using the standard or improved setups.

A critical step during a Dual RNA-Seq experiment is to separate in silico the reads that align to each genome. Another reason to use CLC Genomics Workbench is that after performing the mapping step, the program results in a file containing a list showing in which particular reference the reads are aligned. Based on this list, during a combined analysis, the researcher can easily select and extract all the reads that aligned to each reference genome and save them into separate files (Figure S3). As these files will only contain the reads of one organism, the counting step can be performed using the reference genome and annotations of the corresponding organism.

As a conclusion, with the present work we were able to show that Dual RNA-Seq results vary according to the mapping strategy chosen and this could lead to misinterpretations of the interactions between organisms. Our results showed that the combined analysis allows a smaller loss of reads due to cross-mapping. This fact avoids the loss of relevant information to the first genome chosen in the mapping step when the sequential analysis is used. Since most studies first align the RNA-Seq libraries to the eukaryotic genome, much prokaryotic information is probably being lost. Thus, to fully comprehend gene expression and communication between interacting organisms, we suggest adopting the combined mapping analysis in Dual RNA-Seq experiments.

\section{Acknowledgments}

This work was supported by the Brazilian funding agencies Conselho Nacional de Desenvolvimento Científico e Tecnológico (CNPq), Coordenação de Aperfeiçoamento de Pessoal de Nível Superior (Capes), and by Instituto Nacional de Ciência e Tecnologia da Fixação Biológica do Nitrogênio (INCT-FBN), Brazil.

\section{Conflict of interest}

The authors declare that they have no conflicts of interest related to the subject matter or materials discussed in this article.

\section{Author contributions}

EE and ERS conceived, designed, and performed the experiments. EE, ERS, EB and LMPP analyzed and/or interpreted the data. LMPP contributed to reagents and materials. EE, EB and LMPP wrote the manuscript.

\section{References}

Aprianto R, Slager J, Holsappel S and Veening JW (2016) Timeresolved dual RNA-seq reveals extensive rewiring of lung epithelial and pneumococcal transcriptomes during early infection. Genome Biol 17:198.

Baddal B, Muzzi A, Censini S, Calogero RA, Torricelli G, Guidotti S, Taddei AR, Covacci A, Pizza M, Rappuoli R et al. (2015) Dual RNA-seq of Nontypeable haemophilus influenzae and host cell transcriptomes reveals novel insights into host-pathogen cross talk. MBio 6:e1765-15.

Balsanelli E, Tadra-Sfeir MZ, Faoro H, Pankievicz VCS, de Baura VA, Pedrosa FO, Souza EM, Dixon R and Monteiro RA (2016) Molecular adaptations of Herbaspirillum seropedicae during colonization of the maize rhizosphere. Environ Microbiol 18:2343-2356.

Barret M, Frey-Klett P, Guillerm-Erckelboudt AY, Boutin M, Guernec G and Sarniguet A (2009) Effect of wheat roots infected with the pathogenic fungus Gaeumannomyces graminis var. tritici on gene expression of the biocontrol bacterium Pseudomonas fluorescens Pf29Arp. Mol Plant Microbe Interact 22:1611-1623.

Baruzzo G, Hayer KE, Kim EJ, Di Camillo B, FitzGerald GA and Grant GR (2017) Simulation-based comprehensive benchmarking of RNA-seq aligners. Nat Methods 14:135-139.

Bonato P, Batista MB, Camilios-Neto D, Pankievicz VCS, Tadra-Sfeir MZ, Monteiro RA, Pedrosa FO, Souza EM, Chubatsu LS, Wassem R et al. (2016) RNA-seq analyses reveal insights into the function of respiratory nitrate reductase of the diazotroph Herbaspirillum seropedicae. Environ Microbiol 18:2677-2688.

Boscari A, del Giudice J, Ferrarini A, Venturini L, Zaffini AL, Delledonne M and Puppo A (2013) Expression dynamics of the Medicago truncatula transcriptome during the symbiotic interaction with Sinorhizobium meliloti: which role for nitric oxide? Plant Physiol 161:425-439.

Bruto M, Prigent-Combaret C, Muller D and Moënne-Loccoz Y (2014) Analysis of genes contributing to plant-beneficial functions in plant growth-promoting rhizobacteria and related proteobacteria. Sci Rep 4:6261.

Camilios-Neto D, Bonato P, Wassem R, Tadra-Sfeir MZ, Brusamarello-Santos LCC, Valdameri G, Donatti L, Faoro H, Weiss VA, Chubatsu LS et al. (2014) Dual RNA-seq transcriptional analysis of wheat roots colonized by Azospirillum brasilense reveals up-regulation of nutrient acquisition and cell cycle genes. BMC Genomics 15:378.

Choi YJ, Aliota MT, Mayhew GF, Erickson SM and Christensen BM (2014) Dual RNA-seq of parasite and host reveals gene expression dynamics during filarial worm-mosquito interactions. PLoS Negl Trop Dis 8:e2905.

Faleiro AL, Pereira TP, Espindula E, Brod FCA and Arisi ACM (2013) Real time PCR detection targeting nifA gene of plant growth promoting bacteria Azospirillum brasilense strain FP2 in maize roots. Symbiosis 61:125-133.

Fehr WR, Caviness CE, Burmood DT and Pennington JS (1971) Stage of development descriptions for soybeans, Glycine $\max ($ L.) Merrill1. Crop Sci 11:929-931.

Förstner KU, Vogel J and Sharma CM (2014) READemption-a tool for the computational analysis of deep-sequencing-based transcriptome data. Bioinformatics 30:3421-3423.

Hayden KJ, Garbelotto M, Knaus BJ, Cronn RC, Rai H and Wright JW (2014) Dual RNA-seq of the plant pathogen Phy- 
tophthora ramorum and its tanoak host. Tree Genet Genomes 10:489-502.

Hegedüs Z, Zakrzewska A, Ágoston VC, Ordas A, Rácz P, Mink M, Spaink HP and Meijer AH (2009) Deep sequencing of the zebrafish transcriptome response to mycobacterium infection. Mol Immunol 46:2918-2930.

Hoagland DR and Arnon D (1938) The water culture method for growing plants without soil. Circ Calif Agric Exp Sta 347:32.

Jahn CE, Charkowski AO and Willis DK (2008) Evaluation of isolation methods and RNA integrity for bacterial RNA quantitation. J Microbiol Methods 75:318-324.

Kovalchuk A, Zeng Z, Ghimire RP, Kivimäenpää M, Raffaello T, Liu M, Mukrimin M, Kasanen R, Sun H, Julkunen-Tiitto R et al. (2019) Dual RNA-seq analysis provides new insights into interactions between Norway spruce and necrotrophic pathogen Heterobasidion annosum s.l. BMC Plant Biol 19:2.

Lanubile A, Ferrarini A, Maschietto V, Delledonne M, Marocco A and Bellin D (2014) Functional genomic analysis of constitutive and inducible defense responses to Fusarium verticillioides infection in maize genotypes with contrasting ear rot resistance. BMC Genomics 15:710.

LaMonte GM, Orjuela-Sanchez P, Calla J, Wang LT, Li S, Swann J, Cowell AN, Zou BY, Abdel-Haleem Mohamed AM, Villa Galarce ZH et al. (2019) Dual RNA-seq identifies human mucosal immunity protein Mucin-13 as a hallmark of Plasmodium exoerythrocytic infection. Nat Commun 10:488.

Mateus ID, Masclaux FG, Aletti C, Rojas EC, Savary R, Dupuis C and Sanders IR (2019) Dual RNA-seq reveals large-scale non-conserved genotype $\mathrm{x}$ genotype-specific genetic reprograming and molecular crosstalk in the mycorrhizal symbiosis. ISME J 13:1226-1238.

Mela F, Fritsche K, de Boer W, van Veen JA, de Graaff LH, van den Berg M and Leveau JHJ (2011) Dual transcriptional profiling of a bacterial/fungal confrontation: Collimonas fungivorans versus Aspergillus niger. ISME J 5:1494-1504.

Montoya DJ, Andrade P, Silva BJA, Teles RMB, Ma F, Bryson B, Sadanand S, Noel T, Lu J, Sarno E et al. (2019) Dual RNA-Seq of human letprosy lesions identifies bacterial determinants linked to host immune response. Cell Rep 26:3574-3585.e3.

Mutha NVR, Mohammed WK, Krasnogor N, Tan GYA, Wee WY, Li Y, Choo SW and Jakubovics NS (2019) Transcriptional profiling of coaggregation interactions between Streptococcus gordonii and Veillonella parvula by Dual RNA-Seq. Sci Rep 9:7664.

Oshlack A, Robinson MD and Young MD (2010) From RNA-seq reads to differential expression results. Genome Biol 11:220.

Oya T, Nepomuceno A, Neumaier N, Farias J, Tobita S and Ito O (2004) Drought tolerance characteristics of Brazilian soybean cultivars. Plant Prod Sci 7:129-137.

Packard H, Burke AK, Jensen RV and Stevens AM (2017) Analysis of the in planta transcriptome expressed by the corn pathogen Pantoea stewartii subsp. stewartii via RNA-Seq. PeerJ 5:e3237.

Pankievicz VCS, Camilios-Neto D, Bonato P, Balsanelli E, Tadra-Sfeir MZ, Faoro H, Chubatsu LS, Donatti L, Wajnberg $\mathrm{G}$, Passetti F et al. (2016) RNA-seq transcriptional profiling of Herbaspirillum seropedicae colonizing wheat (Triticum aestivum) roots. Plant Mol Biol 90:589-603.

Reeder SM, Palmer JM, Prokkola JM, Lilley TM, Reeder DM and Field KA (2017) Pseudogymnoascus destructans transcriptome changes during white-nose syndrome infections. Virulence 8:1695-1707.

Schurch NJ, Schofield P, Gierlinski M, Cole C, Sherstnev A, Singh V, Wrobel N, Gharbi K, Simpson GG, Owen-Hughes T et al. (2016) How many biological replicates are needed in an RNA-seq experiment and which differential expression tool should you use? RNA 22:839-851.

Somasegaran P and Hoben HJ (1994) Handbook for Rhizobia: Methods in Legume-Rhizobium Technology. Springer-Verlag, New York.

Thakare D, Yang R, Steffen JG, Zhan J, Wang D, Clark RM, Wang X and Yadegari R (2014) RNA-Seq analysis of laser-capture microdissected cells of the developing central starchy endosperm of maize. Genom Data 2:242-245.

Verwaaijen B, Wibberg D, Kröber M, Winkler A, Zrenner R, Bednarz H, Niehaus K, Grosch R, Pühler A and Schlüter A (2017) The Rhizoctonia solani AG1-IB (isolate 7/3/14) transcriptome during interaction with the host plant lettuce (Lactuca sativa L.). Plos One 12:e177278.

Wang Z, Gerstein M and Snyder M (2009) RNA-Seq: a revolutionary tool for transcriptomics. Nat Rev Genet 10:57-63.

Westermann AJ and Vogel J (2018) Host-Pathogen Transcriptomics by Dual RNA-Seq. In: Arluison V and Valverde C (eds) Bacterial Regulatory RNA - Methods in Molecular Biology. Humana Press, New York, pp 59-75.

Westermann AJ, Gorski SA and Vogel J (2012) Dual RNA-seq of pathogen and host. Nat Rev Microbiol 10:618-630.

Westermann AJ, Förstner KU, Amman F, Barquist L, Chao Y, Schulte LN, Müller L, Reinhardt R, Stadler PF and Vogel J (2016) Dual RNA-seq unveils noncoding RNA functions in host-pathogen interactions. Nature 529:496-501.

Wolf T, Philipp K, Brunke S and Linde J (2018) Two's company: studying interspecies relationships with dual RNA-seq. Curr Opin Microbiol 42:7-12.

\section{Supplementary material}

The following online material is available for this article:

Figure S1 - Filtering procedure and construction of the Chimera library.

Figure S2 - Mapping strategies to determine the number of cross-mapping reads.

Figure S3 Step-by-step of how to extract the reads after the mapping step using the Combined Reference in the CLC workbench environment.

Table S1 - Matrix indicating the reads from (A) Z. mays or (B) Herbaspirillum that were used to determine the true positive (TP), true negative (TN), false positive (FP) and false negative (FN).

Table S2 - Library features and number of total reads attributed to the Herbaspirillum seropedicae or Zea mays genomes according to the mapping approach.

Table S3 - Comparison of the number of reads and where these reads were mapped in each reference genome with the 
mapping parameters of 0.9 of minimum length fraction and 0.8 of minimum similarity fraction.

Table S4 - Comparison of the number of reads incorrectly mapped due to cross-mapping, with the mapping parameters of 0.9 of minimum length fraction and 0.8 of minimum similarity fraction.

Table S5 - Top 20 most counted loci whose reads were lost from the Herbaspirillum (A) and Z. mays (B) libraries.

Table S6 - Top 20 most counted loci whose reads were gained to the Herbaspirillum (A) and Z. mays (B) libraries.
Table S7 - Library features and number of total reads attributed to the Bradyrhizobium elkanii or Glycine max genomes according to the mapping approach.

Table S8 - Library features and number of total reads attributed to the Fusarium verticillioides or Zea mays genomes according to the mapping approach.

Associate Editor: Guilherme Correa de Oliveira

License information: This is an open-access article distributed under the terms of the Creative Commons Attribution License (type CC-BY), which permits unrestricted use, distribution and reproduction in any medium, provided the original article is properly cited. 